\title{
Caracterização de Polímeros e Determinação de Constituintes Inorgânicos em Embalagens Plásticas Metalizadas
}

\author{
Eufemia P. Soares \\ Escola SENAI "Fundação Zerrenner", Escola SENAI Mario Amato, SP \\ Edilene de Cássia D. Nunes \\ Escola SENAI Mario Amato, SP \\ Mitiko Saiki \\ Instituto de Pesquisas Energética e Nucleares, CNEN \\ Hélio Wiebeck \\ Escola Politécnica, USP
}

\begin{abstract}
Resumo: Este trabalho apresenta os resultados analíticos da determinação de elementos químicos em materiais plásticos metalizados, obtidos por análise por ativação com nêutrons (NAA) e os resultados da identificação de polímeros por espectroscopia no infravermelho (IR) e calorimetria exploratória diferencial (DSC). Foram coletadas amostras de plásticos metalizados de embalagens de alimentos e cosméticos. Os resultados dos testes IR e DSC indicaram que os tipos de polímeros usados em plásticos metalizados são polietileno, polipropileno, poli(tereftalato de etileno) e poliestireno. A NAA consistiu em irradiar as amostras e padrões com fluxo de nêutrons térmicos do reator nuclear IEA-R1, seguido da medida da atividade dos raios gama induzidos usando um detector de Germânio hiperpuro acoplado ao espectrômetro de raios gama. Elementos tóxicos como arsênio, cádmio, cromo, níquel e antimônio assim como elementos não tóxicos como bário, cálcio, cobalto, ferro, escândio, selênio e zinco foram determinados e suas concentrações apresentaram grande variabilidade entre as amostras. A análise de polímeros e elementos tóxicos é de grande interesse para o desenvolvimento de processos adequados e seguros para reciclagem e incineração de embalagens plásticas metalizadas, sem causar danos ao ambiente.
\end{abstract}

Palavras-chave: Embalagens, elementos inorgânicos, plásticos metalizados.

\section{Characterization of Polymers and Analysis of Inorganic Constituents in Metallized Plastic Packages}

Abstract: This paper presents analytical results of metallized plastic materials obtained by neutron activation analysis (NAA) and the results of identification of polymers by infrared spectroscopy (IR) and differential scanning calorimetry (DSC). Metallized plastic samples were collected from packages of foodstuffs and cosmetics. Results of IR and DSC tests indicated that polyethylene, polypropylene, poly(ethylene terephthalate) and polystyrene are polymers used in metallized plastics. The NAA consisted of irradiating samples and standards with thermal neutron flux of the IEA-R1 nuclear reactor, followed by induced gamma ray activities measurements using a hyperpure germanium detector coupled to a gamma ray spectrometer. Toxic elements such as arsenic, cadmium, chromium, nickel, and antimony as well as non-toxic elements as barium, calcium, cobalt, iron, scandium, selenium and zinc were determined and their concentrations presented a large variability between the samples. The analyses of polymer and toxic elements are of great interest to develop adequate and safe processes for recycling or incineration of metallized plastic packages without causing damage to the environment.

Keywords: Packages, inorganic elements, metallized plastics.

Autor para correspondência: Eufemia P. Soares, Escola SENAI "Fundação Zerrenner", Rua Serra de Paracaina, 132, CEP: 01522 -020 , Cambuci, São Paulo, SP. E-mail: eufemia_paez@hotmail.com 


\section{Introdução}

Atualmente, os polímeros são aplicados em vários setores, como na construção civil, indústria automotiva, produção de eletro-eletrônicos e embalagens ${ }^{[1]}$. No mercado de embalagens, o plástico ocupa o primeiro lugar entre os maiores produtores de embalagens para o setor alimentício ${ }^{[2]}$. Estas embalagens podem estar nas formas de sacolas, garrafas, caixas e filmes. Os filmes especiais e as embalagens plásticas laminadas (com as suas propriedades de barreira a gases, a vapor d'água, a aromas ou a luz e grande flexibilidade) estão cada vez mais presentes nos supermercados por atenderem às exigências do consumidor, quanto ao acondicionamento e apresentação dos alimentos. Além disso, na indústria de embalagens, a metalização de plásticos vem sendo aplicada para melhorar o acabamento e decorar a peça e, muitas vezes, para melhorar suas propriedades como o aumento da resistência à tração, ao choque e à flexão, melhoria do efeito estético para comercialização, diminuição da absorção de umidade, aumento da tolerância à luz e ao calor, aumento da estabilidade aos produtos químicos e à corrosão ${ }^{[3,4]}$.

Geralmente, peças plásticas metalizadas têm um custo menor que as peças de metal fundidas e, proporcionam economia de peso, maior resistência à corrosão e versatilidade de $\operatorname{projeto}^{[5]}$.

Como conseqüência deste crescente uso de polímeros, em 1995, mais de 600 mil toneladas de lixo plástico (industrial, urbano, rural, entre outros) foram geradas no Brasil e, só a cidade de São Paulo produziu cerca de 12 mil toneladas por dia de lixo sólido, sendo 700 toneladas de embalagens plásticas ${ }^{[6]}$. Na caracterização físico-química dos resíduos sólidos domiciliares do município de São Paulo, feita em maio de 1998 observou-se que cerca de $22,9 \%$ deste resíduo é de material plástico (cerca de 2000 toneladas), um aumento significativo em relação aos anos anteriores ${ }^{[7]}$.

Desta forma, o destino final destes materiais constitui uma das grandes preocupações da sociedade atual. Os plásticos degradam-se muito lentamente no ambiente. Uma vez que eles são bastante resistentes às radiações, ao calor, ao ar e à água. Representam cerca de 6 a $7 \%$ em peso e $16 \%$ em volume nos resíduos sólidos urbanos ${ }^{[8]}$ e a maior parte do resíduo plástico descartado permanece nos vazadouros ou lixões ${ }^{[6]}$. O desenvolvimento industrial e populacional agravou este problema, exigindo soluções para dimi- nuir o impacto no ambiente. Parte destes plásticos podem ser recuperados pela reciclagem mecânica, produzindo novos materiais, normalmente com usos menos nobres, como por exemplo na construção civil e nas rodovias, e nos materiais para sinalização de estradas. Também os plásticos podem ser incinerados produzindo energia ${ }^{[9]}$. Porém nesta reciclagem energética pode ocorrer a formação de gases tóxicos, volatilização de metais pesados, ou ainda cinzas contendo elementos tóxicos. Portanto, esta prática poderá ser feita somente sob controle rigoroso.

Face ao exposto, o objetivo do presente trabalho foi determinar os constituintes inorgânicos presentes em amostras de embalagens plásticas metalizadas bem como caracterizar os tipos de polímeros nessas embalagens. Estas determinações são de grande interesse na seleção de plásticos para reciclagem, incineração ou para um tratamento e aproveitamento destes materiais, de maneira segura, sem ocasionar danos ao ambiente. Os elementos foram analisados pelo método instrumental de análise por ativação com nêutrons (NAA) e a caracterização dos polímeros foi feita por espectrometria no infravermelho (IR) e calorimetria exploratória diferencial (DSC).

\section{Experimental}

\section{Amostras e seu Tratamento para Análise}

As amostras plásticas metalizadas adquiridas foram embalagens de alimentos e cosméticos (batons, perfumes, entre outras). As embalagens de alimentos receberam o código $\mathrm{AE}$ e as de cosméticos, o código $\mathrm{CP}$.

Para NAA as embalagens foram cortadas em pedaços com dimensões de cerca de $1 \mathrm{~cm}$ x $1 \mathrm{~cm}$, usando um par de tesouras de aço inoxidável. Estas amostras foram lavadas com água deionizada e em seguida foram esfregadas com chumaços de algodão embebidos com hexano p.a. Para irradiação no reator as amostras foram pesadas em invólucros de polietileno de alta pureza e a massa de cada amostra utilizada foi de cerca de $100 \mathrm{mg}$.

\section{Preparação de Padrões de Elementos para Uso na NAA}

Prepararam-se padrões sintéticos simples e multielementares a partir de soluções padrão. Estes padrões sintéticos foram preparados sobre papel de filtro, com as seguintes massas, em $\mu \mathrm{g}$ : arsênio (As) $=$ 1,50 ; bário $(\mathrm{Ba})=49,9$; bromo $(\mathrm{Br})=5,098$; cálcio 
$(\mathrm{Ca})=501,70$; cádmio $(\mathrm{Cd})=3,507$; cobalto $(\mathrm{Co})=$ 0,100 ; cromo $(\mathrm{Cr})=1,497$; ferro $(\mathrm{Fe})=501,70$; níquel $(\mathrm{Ni})=50,15$; antimônio $(\mathrm{Sb})=0,601$; escândio $(\mathrm{Sc})=$ 0,0601 ; selênio $(\mathrm{Se})=8,0$ e zinco $(\mathrm{Zn})=34,608$.

\section{Procedimento para Análise de Constituintes Inorgânicos}

O método de ativação com nêutrons aplicado na análise de plásticos consistiu em irradiar as amostras e os padrões elementares no reator IEA-R1 por um período de 16 horas sob um fluxo de nêutrons térmicos de $10^{12} \mathrm{ncm}^{-2} \mathrm{~s}^{-1}$. Após tempos de decaimento adequados, as amostras e os padrões sintéticos foram montados em pranchetas (panelinhas) de aço inoxidável para a medida de atividades gama. A primeira medida foi efetuada após uma média de 5 dias de decaimento e a segunda contagem após uma média de 10 dias de decaimento. Cada padrão foi medido por um período de 5400 a 6000 segundos e cada amostra de 25000 a 50000 segundos. As medidas foram feitas no sistema Canberra, utilizando detector de Ge hiperpuro (modelo GX2020), que está acoplado ao Processador integrado de sinais modelo 1510 e a placa S100, ambos da Canberra. A resolução do sistema (FWHM) utilizado foi de $0,80 \mathrm{keV}$ para o fotopico $121,97 \mathrm{keV}$ de ${ }^{57} \mathrm{Co}$ e $1,80 \mathrm{keV}$ para fotopico de $1331,57 \mathrm{keV}$ da fonte de ${ }^{60} \mathrm{Co}$.

Para a aquisição dos espectros utilizou-se o programa S100 da Canberra e para processamento dos dados de contagem o programa VERSAO2, uma nova versão do programa VISPECT2. A identificação dos radioisótopos foi feita pela meia vida e energia dos raios gama. As concentrações dos elementos foram calculadas pelo método comparativo, aplicando-se a relação ${ }^{[10]}$ :

$$
\mathrm{C}_{\mathrm{a}}=\left\{\mathrm{m}_{\mathrm{p}} \mathrm{A}_{\mathrm{a}} \mathrm{e}^{0,693\left(\mathrm{t}_{\mathrm{da}}-\mathrm{t}_{\mathrm{d} p}\right) / \mathrm{t}_{1 / 2}}\right\} /\left[\mathrm{A}_{\mathrm{p}} \mathrm{M}_{\mathrm{a}}\right]
$$

Nesta equação, os índices a e p referem-se à amostra e padrão, respectivamente; $\mathrm{M}_{\mathrm{a}}=$ massa total de amostra; $\mathrm{C}_{\mathrm{a}}=$ concentração do elemento na amos$\operatorname{tra}\left(\mathrm{C}_{\mathrm{a}}=\mathrm{m}_{\mathrm{a}} \mathrm{M}_{\mathrm{a}}\right) ; \mathrm{t}_{\mathrm{d}}=$ tempo de decaimento; $\mathrm{m}_{\mathrm{p}}=$ massa do elemento padrão; $\mathrm{A}=$ taxa de contagens; $\mathrm{t}_{1 / 2}=$ tempo de meia vida do radioisótopo medido.

Para avaliar a exatidão e a precisão dos resultados da NAA foram analisados os materiais certificados de referência: NIST 1577b Bovine Liver SRM 1577b do National Institute of Standards and Technology, USA ${ }^{[11]}$, BCSS-1 Marine Sediment proveniente do National Research Council Canadá ${ }^{12]}$ e Polietileno VDA 002, VDA 003 e IMEP-10 do Institute for Reference Materials and Measurements, Comission of the
European Communities ${ }^{[13]}$. As condições experimentais utilizadas na análise dos materiais de referência foram as mesmas das análises das amostras de plástico com exceção do tempo de irradiação que foi de 8 horas. Para expressar os resultados das análises do material Bovine Liver foi determinada a porcentagem de perda em massa na secagem e o valor obtido foi de $4,53 \%$.

Parte dos resultados da exatidão e precisão obtidos pelo procedimento experimental de NAA adotado foram apresentados no trabalho anterior ${ }^{[14]}$.

\section{Procedimento para Análise por Espectrometria no Infravermelho}

Para identificar o polímero por IR foi necessário separar o material plástico do metal. Para isso, baseando-se nos dados de resistência química de plásticos a solventes apresentados por Agnelli[15], as amostras foram mergulhadas em soluções concentradas de ácido nítrico ou clorídrico por um tempo suficiente para eliminação do metal por dissolução, sem afetar o polímero. Em seguida estas amostras isentas da parte metalizada foram lavadas em água deionizada e submetidas a secagem.

O estado físico de cada amostra, definiu o seu modo de preparação, bem como o tipo de acessório a ser utilizado. As amostras que se apresentaram na forma de filmes transparentes foram analisadas diretamente no espectrofotômetro IR. Para o caso das amostras de embalagens de cosméticos foi feita, ou a dissolução destas amostras em solventes orgânicos como clorofórmio, metil etil cetona ou acetato de etila, ou pirólise delas, e o filme ou vapor formado foi recolhido sobre cristal de $\mathrm{KBr}$ e analisado.

O espectrofotômetro utilizado foi do tipo FTIR, marca Nicolet Magna IR 550 series II, com resolução de $4,0 \mathrm{~cm}$, divisor de feixe de $\mathrm{KBr}$, detetor DTGS/ $\mathrm{KBr}$ e número de varreduras de 64.

O espectro foi obtido utilizando-se o programa OMNIC versão 4.1 e para obtenção das bandas de absorção utilizou-se a função "find peaks". A identificação dos polímeros foi feita por comparação dos espectros das amostras com espectros padrão contidos na biblioteca Hummel Polymer and Additives ${ }^{[16]}$. Após a comparação com os espectros da biblioteca de espectros procedeu-se à interpretação das bandas de absorção.

\section{Procedimento para Análise por Calorimetria Exploratória Diferencial}

As amostras foram limpas com água deionizada e hexano e secas a temperatura ambiente. Cada amos- 
tra foi cortada em pedaços de $5 \mathrm{~mm} \times 5 \mathrm{~mm}$ ( 5 a 10 $\mathrm{mg}$ ), e submetida à análise no analisador térmico modelo DSC 910 da TA Instruments, com taxa de aquecimento de $10{ }^{\circ} \mathrm{C} / \mathrm{min}$, para faixa de temperatura de 20 a $350{ }^{\circ} \mathrm{C}$.

Na identificação, os picos obtidos nas curvas DSC das amostras foram comparados com aqueles apresentados por Brandup ${ }^{[17]}$.

\section{Resultados e Discussão}

Os resultados das análises de arsênio, bário, bromo, cálcio, cádmio, cobalto, cromo, ferro, níquel, antimônio, escândio, selênio e zinco em embalagens plásticas metalizadas de diferentes tipos de alimentos e cosméticos são apresentados nas Tabelas 1 e 2. As concentrações destes elementos variaram de níveis de $\mu \mathrm{g} \mathrm{kg}^{-1}$ a $\mu \mathrm{g} \mathrm{g}^{-1}$. Observa-se nestas Tabelas que para algumas amostras nem todos os elementos foram detectados, devido às suas baixas concentrações ou interferências na análise. Os elementos bromo, cobalto, cromo, antimônio, escândio e zinco foram encontrados em todas as amostras de embalagens analisadas. O elemento tóxico arsênio foi encontrado em algumas amostras de embalagens em teores menores que $19 \mu \mathrm{g} \mathrm{kg}^{-1}$ e os teores de cromo variaram de 0,12 a $163 \mu \mathrm{g} \mathrm{g}^{-1}$. Foram detectados, também, cádmio e níquel em algumas amostras.

Tabela 1. Concentrações de alguns elementos químicos nas amostras de embalagens de alimentos $-\mathrm{AE}$

\begin{tabular}{|c|c|c|c|c|c|}
\hline \multirow{2}{*}{ Elemento } & \multicolumn{5}{|c|}{ Embalagem plástica metalizada de alimentos } \\
\hline & AE1 & AE2 & AE3 & AE4 & AE5 \\
\hline As $\mu \mathrm{g} \mathrm{kg}^{-1}$ & -* & $139 \pm 6$ & $191 \pm 4$ & - & - \\
\hline $\mathrm{Ba} \mu \mathrm{g} \mathrm{g}^{-1}$ & - & $35 \pm 4$ & $280 \pm 5$ & - & - \\
\hline $\mathrm{Br} \mu \mathrm{g} \mathrm{kg}^{-1}$ & $552 \pm 15^{* * *}$ & $682 \pm 8$ & $1649 \pm 68$ & $1555 \pm 68$ & $1752 \pm 14$ \\
\hline $\mathrm{Ca} \mu \mathrm{g} \mathrm{g}^{-1}$ & - & $306 \pm 77$ & - & - & - \\
\hline $\mathrm{Cd} \mu \mathrm{g} \mathrm{kg}^{-1}$ & - & $985 \pm 142$ & - & - & - \\
\hline Co $\mu \mathrm{g} \mathrm{kg}^{-1}$ & $14 \pm 7$ & $179 \pm 5$ & $24 \pm 3$ & $230 \pm 6$ & $3922 \pm 49$ \\
\hline $\mathrm{Cr} \mu \mathrm{g} \mathrm{g}^{-1}$ & $0,24 \pm 0,03$ & $163 \pm 2$ & $2,22 \pm 0,09$ & $0,17 \pm 0,07$ & $1,44 \pm 0,04$ \\
\hline $\mathrm{Fe} \mu \mathrm{g} \mathrm{g}^{-1}$ & $12,1 \pm 0,9$ & - & $24,7 \pm 1,8$ & - & $918 \pm 7$ \\
\hline Ni $\mu \mathrm{g} \mathrm{g}^{-1}$ & - & - & $1,9 \pm 0,7$ & - & $13,1 \pm 1,3$ \\
\hline $\mathrm{Sb} \mu \mathrm{g} \mathrm{kg}^{-1}$ & $7,2 \pm 0,6$ & $36297 \pm 113$ & $17116 \pm 55$ & $44346 \pm 140$ & $54,4 \pm 1,4$ \\
\hline $\mathrm{Sc} \mu \mathrm{g} \mathrm{kg}^{-1}$ & $2,1 \pm 0,2$ & $129,4 \pm 0,7$ & $135,5 \pm 0,7$ & $74,2 \pm 0,7$ & $22,4 \pm 0,7$ \\
\hline Se $\mu \mathrm{g} \mathrm{kg}^{-1}$ & $38 \pm 10$ & $3591 \pm 85$ & $2188 \pm 68$ & $3158 \pm 89$ & $87 \pm 28$ \\
\hline $\mathrm{Zn} \mu \mathrm{g} \mathrm{g}^{-1} 1$ & $640 \pm 3$ & $10,1 \pm 0,2$ & $23,1 \pm 0,3$ & $13,7 \pm 0,2$ & $1088 \pm 5$ \\
\hline
\end{tabular}

*o símbolo "-_ significa elemento químico não detectado

**A incerteza foi calculada usando os erros de medidas das taxas de contagem
Tabela 2. Concentrações de alguns elementos químicos nas amostras de embalagens de cosméticos - CP

\begin{tabular}{lccccc}
\hline \multirow{2}{*}{ Elemento } & \multicolumn{5}{c}{ Embalagens plásticas metalizadas de cosméticos } \\
\cline { 2 - 6 } & $\mathrm{CP} 2$ & $\mathrm{CP} 3$ & $\mathrm{CP} 4$ & $\mathrm{CP} 5$ & $\mathrm{CP} 6$ \\
\cline { 2 - 6 } As $\mu \mathrm{g} \mathrm{kg}^{-1}$ & $5,9 \pm 0,6$ & $194 \pm 25$ & $1,4 \pm 0,6$ & $4,4 \pm 0,5$ & $5,0 \pm 1,1$ \\
$\mathrm{Ba} \mu \mathrm{g} \mathrm{g}^{-1}$ & - & $14,2 \pm 1,2$ & - & - & - \\
$\mathrm{Br} \mu \mathrm{g} \mathrm{kg}^{-1}$ & $359 \pm 7$ & $120 \pm 3$ & $95 \pm 4$ & $315 \pm 5$ & $630 \pm 40$ \\
$\mathrm{Ca} \mu \mathrm{g} \mathrm{g}^{-1}$ & $158 \pm 23$ & $254 \pm 32$ & $208 \pm 16$ & $134 \pm 14$ & - \\
$\mathrm{Cd} \mu \mathrm{kg}^{-1}$ & $29 \pm 9$ & $40 \pm 17$ & - & - & - \\
$\mathrm{Co} \mu \mathrm{gg}^{-1}$ & $2592 \pm 38$ & $79 \pm 3$ & $18 \pm 1$ & $38 \pm 2$ & $3278 \pm 32$ \\
$\mathrm{Cr} \mu \mathrm{g} \mathrm{g}^{-1}$ & $0,98 \pm 0,02$ & $0,12 \pm 0,03$ & $0,121 \pm 0,009$ & $2,59 \pm 0,03$ & $5,18 \pm 0,05$ \\
Fe $\mu \mathrm{g} \mathrm{g}^{-1}$ & $58 \pm 2$ & $7,0 \pm 1,9$ & $6,4 \pm 0,7$ & $18,6 \pm 1,2$ & $19,6 \pm 1,5$ \\
Sb $\mu \mathrm{g} \mathrm{g}^{-1}$ & $15,0 \pm 0,5$ & $76,6 \pm 1,5$ & $3,82 \pm 0,29$ & $8,0 \pm 0,2$ & $17,5 \pm 1,0$ \\
Sc $\mu \mathrm{g} \mathrm{kg}^{-1}$ & $1,4 \pm 0,2$ & $45,5 \pm 0,5$ & $0,45 \pm 0,09$ & $5,0 \pm 0,1$ & $19,8 \pm 0,2$ \\
Se $\mu \mathrm{g} \mathrm{kg}^{-1}$ & - & $2230 \pm 35$ & - & - & $36 \pm 11$ \\
Zn $\mu \mathrm{g} \mathrm{g}^{-1} 1$ & $10,4 \pm 0,2$ & $152,3 \pm 1,0$ & $14,5 \pm 0,2$ & $3,7 \pm 0,9$ & $107,6 \pm 0,5$ \\
\hline
\end{tabular}

No trabalho anterior ${ }^{[14]}$ utilizaram-se os materiais de referência NIST $1577 b$ Bovine Liver ${ }^{[11]}$ e NRCC BCSS-1 Marine Sediment ${ }^{[12]}$, e cujas médias dos resultados para arsênio, bromo, cobalto, cromo, ferro, níquel, selênio e zinco são apresentadas na Tabela 3. Na Tabela 4, estão os resultados dos materiais de referência IRMM - Polietileno VDA002, VDA003 e IMEP-10 ${ }^{[13]}$.

Os resultados dos materiais de referência do NIST e NRCC apresentam, em geral, uma boa concordância com os valores certificados com porcentagem de erros relativos inferiores a $12,6 \%$ e boa precisão com desvios padrão relativos variando de 0,25 a 1,7 \%. Resultados menos exatos foram obtidos para os elementos níquel e selênio devido às baixas taxas de contagens obtidos na medida do cobalto-58 e do selênio-75. Os materiais de referência IRMM, apresentam resultados com erros relativos menores que $10 \%$ e desvios padrão relativos de 0,13 a $8,9 \%$.

Para os resultados obtidos nas Tabelas 3 e 4 foram calculados os valores de diferenças padronizadas ou z-score aplicando-se a relação ${ }^{[18]}$.

$$
\mathrm{z}-\mathrm{score}=\frac{(\mathrm{Ci}-\mathrm{Cr})}{\sqrt{\left(\mathrm{s}_{\mathrm{i}}\right)^{2}+\left(\mathrm{s}_{\mathrm{r}}\right)^{2}}}
$$

sendo: $\mathrm{C}_{\mathrm{i}}=$ resultado obtido para o elemento $\mathrm{i}$; $\mathrm{C}_{\mathrm{r}}=$ valor certificado; $\mathrm{s}_{\mathrm{i}}=$ desvio-padrão do resultado obtido; $\mathrm{s}_{\mathrm{r}}=$ desvio padrão do valor certificado.

Os resultados das Tabelas 3 e 4 indicam que os resultados obtidos para os valores $\mathrm{z}$ variaram de $-1,69$ a 1,18 e estão dentro da faixa dos valores do certifica- 
Tabela 3. Resultados da Análise de materiais certificados (resultados em $\mu \mathrm{g} \mathrm{g}^{-1}$ )

\begin{tabular}{|c|c|c|c|c|c|c|}
\hline Elemento & $\mathbf{M R}^{(\mathbf{a})}$ & Este Trabalho $\mathbf{x} \pm \mathbf{S}^{(\mathrm{b})}$ & $\begin{array}{c}\mathbf{E r}^{(\mathbf{c})} \\
\%\end{array}$ & $\begin{array}{c}\mathrm{Sr}^{(\mathrm{d})} \\
\%\end{array}$ & Valores certificados & z-score \\
\hline \multirow{2}{*}{ As } & (1) & $0,040 \pm 0,009$ & - & - & $(0,05)^{(\mathrm{e})}$ & - \\
\hline & (2) & $11,4 \pm 0,2$ & 3,0 & 1,7 & $11,1 \pm 1,4$ & 0,212 \\
\hline $\mathrm{Br}$ & (1) & $10,46 \pm 0,07$ & - & - & $(9,7)$ & - \\
\hline \multirow{2}{*}{$\mathrm{Co}$} & (1) & $0,301 \pm 0,006$ & - & - & $(0,25)$ & - \\
\hline & (2) & $12,01 \pm 0,09$ & 5,3 & 0,75 & $11,4 \pm 2,1$ & 0,290 \\
\hline $\mathrm{Cr}$ & (2) & $138,6 \pm 0,8$ & 13 & 0,58 & $123 \pm 14$ & 1,112 \\
\hline \multirow{2}{*}{$\mathrm{Fe}$} & (1) & $169 \pm 4$ & 8,2 & 2,4 & $184 \pm 15$ & $-0,966$ \\
\hline & (2) & $33417 \pm 85$ & 1,6 & 0,25 & $32900 \pm 980$ & 0,526 \\
\hline $\mathrm{Ni}$ & (2) & $70 \pm 6$ & 27 & 8,6 & $55,3 \pm 3,6$ & 2,101 \\
\hline $\mathrm{Se}$ & (1) & $0,88 \pm 0,03$ & 20 & 3,4 & $0,73 \pm 0,06$ & 2,236 \\
\hline \multirow{2}{*}{$\mathrm{Zn}$} & (1) & $119,0 \pm 0,5$ & 6,7 & 0,42 & $127 \pm 16$ & $-0,500$ \\
\hline & (2) & $125 \pm 1$ & 4,8 & 0,80 & $119 \pm 12$ & 0,498 \\
\hline
\end{tabular}

(a) MR =- Material de referência - (1) NIST 1577b Bovine Liver; (2) NRCC - BCSS-1 Marine Sediment

(b) $\mathrm{x} \pm \mathrm{s}$ - média aritmética e desvio padrão.

(c) $\mathrm{E}_{\mathrm{r}}$ - Erro relativo percentual

(d) $\mathrm{S}_{\mathrm{r}}$ - desvio padrão relativo

(e) Números entre parênteses indicam valores informativos

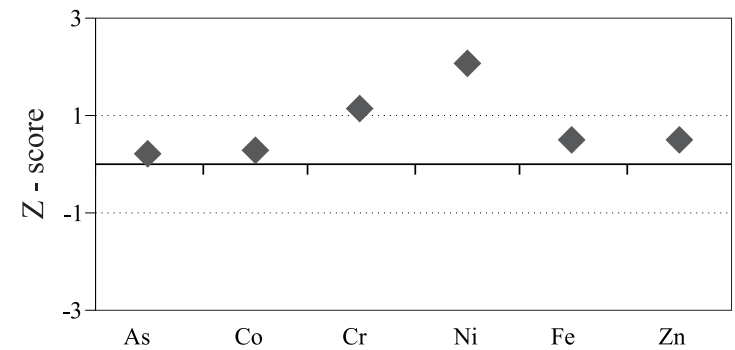

Figura 1. Valores de z para arsênio, cobalto, cromo, ferro e zinco no material de referência Bovine Liver

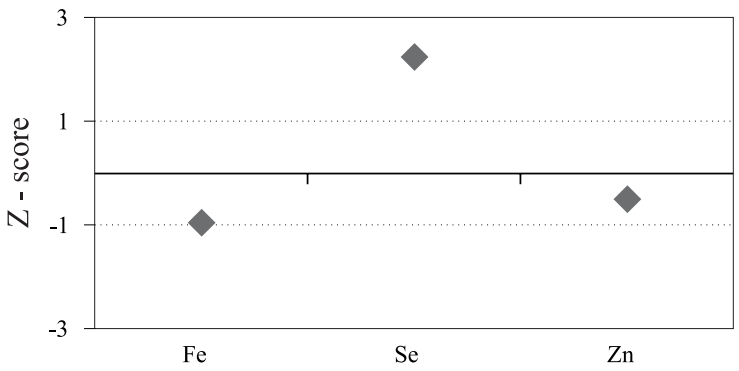

Figura 2. Valores de z para ferro, selênio e zinco no material de referência Marine Sediment

Tabela 4. Resultados da Análise de materiais de referência de polietileno (resultados em $\mu \mathrm{g} \mathrm{g}^{-1}$ )

\begin{tabular}{|c|c|c|c|c|c|c|}
\hline Elemento & $\mathbf{M R}^{(\mathrm{a})}$ & Este Trabalho $\mathbf{x} \pm \mathbf{S}^{(\mathbf{b})}$ & $\begin{array}{c}\mathbf{E r}^{(\mathrm{c})} \\
\%\end{array}$ & $\begin{array}{c}\mathrm{Sr}^{(\mathrm{d})} \\
\%\end{array}$ & Valores certificados & z-score ${ }^{(6)}$ \\
\hline As & (5) & $9,45 \pm 0,08$ & 1,6 & 0,85 & $9,61 \pm 0,57$ & $-0,274$ \\
\hline $\mathrm{Br}$ & (5) & $308,4 \pm 0,5$ & 10 & 0,18 & $280 \pm 24$ & 1,184 \\
\hline \multirow{3}{*}{$\mathrm{Cd}$} & (3) & $72,9 \pm 0,3$ & 4,0 & 0,13 & $75,9 \pm 2,1$ & $-1,427$ \\
\hline & (4) & $197,8 \pm 0,8$ & 3,1 & 0,41 & $191,9 \pm 4,8$ & 1,220 \\
\hline & (5) & $108,9 \pm 1,0$ & 9,0 & 8,9 & $119,72 \pm 6,29$ & $-1,692$ \\
\hline $\mathrm{Cr}$ & (5) & $95,6 \pm 1,7$ & 5,2 & 1,8 & $100,9 \pm 5,7$ & $-0,891$ \\
\hline
\end{tabular}

(a) MR =- Material de referência - (3) IRMM - Polietileno VDA002; (4) IRMM - Polietileno VDA003; (5) IRMM - Polietileno IMEP-10

(b) $\mathrm{x} \pm \mathrm{s}$ - média aritmética e desvio padrão.

(c) $\mathrm{E}_{\mathrm{r}}$ - Erro relativo percentual

(d) $\mathrm{S}_{\mathrm{r}}$ - desvio padrão relativo 
Tabela 5. Identificação dos polímeros em embalagens plásticas de alimentos (AE) e de cosméticos (CP)

\begin{tabular}{|c|c|c|}
\hline \multirow{2}{*}{ Amostras } & IR & DSC* \\
\hline & Polímeros** & Polímeros (Temperatura; $\mathrm{Tm} / \mathrm{Tg}, \mathrm{em}^{\circ} \mathrm{C}$ ) \\
\hline AE1 & PP; PE & $\operatorname{LDPE}(\mathrm{Tm}=99) ; \operatorname{HDPE}(\mathrm{Tm}=131) ; \mathrm{PP}(\mathrm{Tm}=166)$ \\
\hline AE2 & PE; PET & LDPE $(\mathrm{Tm}=109) ; \quad$ PE $(\mathrm{Tm}=124) ;$ PET $(\mathrm{Tm}=251)$ \\
\hline AE3 & PP & HDPE $(\mathrm{Tm}=130) ; \quad$ PP $(\mathrm{Tm}=169)$ \\
\hline AE4 & PE; PET & PE $(\mathrm{Tm}=125) ; \quad$ PET $(\mathrm{Tm}=251) ; \operatorname{LDPE}(\mathrm{Tm}=110)$ \\
\hline AE5 & $\mathrm{PE}$ & $\operatorname{LDPE}(\mathrm{Tm}=101)$ \\
\hline $\mathrm{CP} 2$ & PP & $\mathrm{PP}(\mathrm{Tm}=166)$ \\
\hline $\mathrm{CP} 3$ & $\mathrm{PP}$ & $\mathrm{PP}(\mathrm{Tm}=166)$ \\
\hline $\mathrm{CP} 4$ & $\mathrm{PP}$ & $\mathrm{PP}(\mathrm{Tm}=164)$ \\
\hline $\mathrm{CP} 5$ & $\mathrm{PP}$ & $\mathrm{PP}(\mathrm{Tm}=166)$ \\
\hline CP6 & PS & PS $(\mathrm{Tg}=95)$ \\
\hline
\end{tabular}

* Resultados com mais de um tipo de polímero referem-se a filmes multicamadas

**polietileno de alta (HDPE), média (PE) e baixa densidade (LDPE); polipropileno (PP); poli(tereftalato de etileno) (PET); poliestireno (PS).

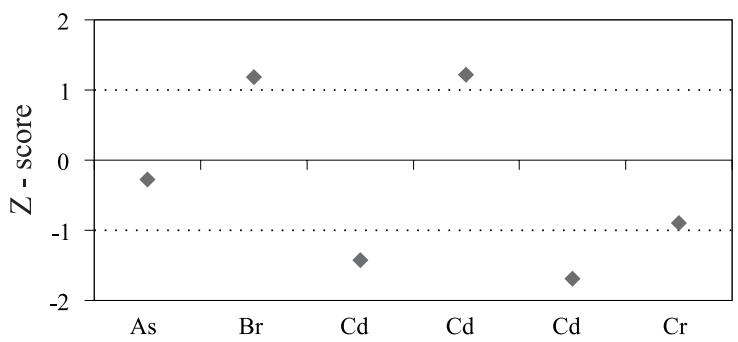

Figura 3. Valores z para arsênio, bromo, cádmio e cromo no material de referência Polietileno VDA002, VDA003 e IMEP-10

do a um nível de $99 \%$ de confiança ${ }^{[18]}$. Estes resultados podem ser observados nas Figuras 1, 2 e 3.

Resultados das análises por espectrometria no infravermelho e calorimetria de varredura diferencial

Os resultados da identificação dos polímeros por IR e DSC estão apresentados na Tabela 5. Nas embalagens de alimentos foram identificados polietileno de baixa, média e alta densidade, polipropileno e poli(tereftalato de etileno). Nas embalagens de cosméticos foram identificados polipropileno e poliestireno.

\section{Conclusões}

A NAA foi adequada para a caracterização dos componentes inorgânicos em plásticos metalizados devido ao seu caráter multielementar para análise de uma ampla faixa de concentrações e principalmente devido à não necessidade de dissolução das amostras que, no caso de plásticos é bastante difícil. Concluiuse também que certos tipos de embalagens plásticas metalizadas podem constituir uma possível fonte de contaminação ambiental de elementos tóxicos como cádmio, níquel e antimônio. As análises por IR e DSC foram importantes na caracterização dos polímeros utilizados na confecção de embalagens metalizadas e também para o desenvolvimento de um processo seguro para a reciclagem ou incineração de plásticos metalizados para que não ocorra a contaminação do meio ambiente.

\section{Agradecimentos}

Os autores agradecem à Escola SENAI Mario Amato, Laboratório DuPont/SENAI, à FAPESP e ao CNPq.

\section{Referências Bibliográficas}

1. Spieth, E.H. - "Mercado brasileiro de plásticos", in: Anais do I Congresso Brasileiro de Polímeros, Associação Brasileira de Polímeros, p.30 -34, São Paulo-SP, nov (1991).

2. Sino, M.A. - Plástico Moderno, 254, p.12-22 (1995).

3. Sarantópoulos, C.I.G.L.; Oliveira, L.M.M. - "The impact of plastics packages on the food industry", in: 
Anais do $2^{\circ}$ Congresso Brasileiro de Polímeros, p.7379, São Paulo-SP, out (1993).

4. Sino, M.A. - Plástico moderno, 282, p.24-31 (1997).

5. Wiebeck, H.; Harada, J.; Araújo, S.C., “Acabamentos em artefatos de plástico”, CECAE/USP, São Paulo, SP, (s.d).

6. Wiebeck, H.; Piva, A.N.; Risso, W.M.; , Angelatakis, A.R.; Schwarz, L.B.; Soares, M.A. - "Dificuldades na reciclagem do plástico do resíduo sólido urbano do lixão", in: Anais do $3^{\circ}$ Congresso Brasileiro de Polímeros, v.2, p.1183-1186, RJ, out/nov (1995).

7. Orth, M.H.A.; Motta, F. S.- Limpeza Pública, 48, p.916 (1998).

8. CEMPRE, Perfil da recicladora de plástico, RJ, (1994).

9. Ortiz, S.A. - "A embalagem e o meio ambiente", in: Anais do Seminário Internacional Indústria e Meio Ambiente: Reciclagem do Lixo e Controle da Poluição, v. II, p.254-261 (1992).

10. De Soete, D.; Gilbels, R.; Hoste, J. - "Neutron activation analysis", Wiley-Interscience, NY, (1972).

11. NIST - National Institute of Standards and Technology, "Certificate of analysis standard reference material 1577b bovine liver”, (1991).
12. NRC - National Research Council, Canada, "Certified values for BCSS-1 Marine Sediment”, (1987).

13. IRMM - Institute for Reference Materials and Measurements, Commission of the European Communities, Joint Research Centre - "Polyethylene VDA 002/ VDA 003/ IMEP-10 - Certified materials for determination of cadmium and trace elements in polyethylene" (1993).

14. Soares, E.P.; Saiki, M.; Wiebeck, H. - "Análise de elementos químicos e tipos de polímeros em materiais plásticos metalizados", in: Anais do $56^{\circ}$ Congresso Brasileiro de Metalurgia, p.601-611, Belo Horizonte-MG, jul (2001).

15. Agnelli, J.A.M. - "Características especiais de polimeros", UFSCar/UNESP (1999).

16. Nicolet - "Hummel Polymer Library." Version 1.02. Nicolet Inst. Corp. (1985).

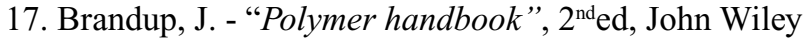
and Sons, NY (1989).

18. Bode, P. - "Instrumental and organizational aspects of a neutron activation analysis laboratory", Tese de Doutorado, Interfaculty Reactor Institut, The Netherlands (1996).

Recebido: $10 / 08 / 01$

Aprovado: 02/07/02 